This item was submitted to Loughborough's Research Repository by the author.

Items in Figshare are protected by copyright, with all rights reserved, unless otherwise indicated.

\title{
Image fusion based multi resolution and frequency partition discrete cosine transform for palm vein recognition
}

PLEASE CITE THE PUBLISHED VERSION

https://doi.org/10.1109/IEA.2019.8715136

PUBLISHER

(C) IEEE

VERSION

AM (Accepted Manuscript)

LICENCE

CC BY-NC-ND 4.0

\section{REPOSITORY RECORD}

Soh, Shi C., M.Z. Ibrahim, M.F. Abas, N. Sulaiman, and David J. Mulvaney. 2019. "Image Fusion Based Multi Resolution and Frequency Partition Discrete Cosine Transform for Palm Vein Recognition”. figshare. https://hdl.handle.net/2134/38335. 


\section{Image fusion based Multi Resolution and Frequency Partition Discrete Cosine Transform for palm vein recognition}

\author{
Shi Chuan Soh and M. Z. Ibrahim* \\ Faculty of Electrical and Electronic Engineering \\ Universiti Malaysia Pahang \\ 26600 Pekan, Pahang, Malaysia \\ e-mail: sschuan92@gmail.com,zamri@ump.edu.my
}

\author{
D. J. Mulvaney \\ School of Electronic, Electrical and Systems \\ Engineering, \\ Loughborough University \\ LE11 3TU, United Kingdom \\ e-mail: d.j.mulvaney@lboro.ac.uk
}

\begin{abstract}
The rapid growth of technology has increased the demand for automated security systems. Due to the accessibility of the palm region and the unique characteristics of each individual's palm vein features, such biometrics have been receiving particular attention. In the published research relating to palm vein biometrics, usually only a single image is used to supply the data for recognition purposes. Previous experimental work has demonstrated that the fusion of multiple images is able to provide richer feature information resulting in an improved classification performance. However, although most of the image fusion techniques are able to preserve the vein pattern, the fused image is often blurred, the colors are distorted and the spatial resolution reduced. In this paper, the multiresolution discrete cosine transform (MRDCT) and frequency partition DCT (FPDCT) image fusion are applied and are able to extract the finer details of vein patterns while reducing the presence of noise in the image. The performance shows that the use of MRDCT and FPDCT was able to improve recognition rate compared to using a single image. The equal error rate improvement is also significant, falling to $9 \%$ in $700 \mathrm{~nm}$ image, $7 \%$ in $850 \mathrm{~nm}$ image and $6 \%$ in $940 \mathrm{~nm}$ image.
\end{abstract}

Keywords-palm vein; image fusion; multi-resolution; frequency partition; dct

\section{INTRODUCTION}

Biometrics can play a crucial role in protecting personal information and the prevention of identity theft. Current identification systems still use ID cards, passwords or security numbers that risk being forgotten, forged or stolen. These methods are now being gradually replaced by biometric methods that capture an individual's physical and behavioral characteristics and so reduce the risk of fraud arising due to forgery or theft. A number of different characteristics have been used for biometrics, such as fingerprints, voice, veins or signature patterns.

Vein patterns have been found to be unique to individuals and are sufficiently distinctive that even the vein patterns of identical twins can be distinguished. Consequently, vein recognition is a promising method for identifying individuals for security purposes and has become a popular approach for investigation by a number of researchers. Palm veins are difficult to forge as they are found underneath the skin, recognition can be formulated to be robust to axial and scales changes and can be implemented in a contactless manner thereby reducing the possibility of cross-contamination.

In the last two decades, considerable research has been carried out to investigate the potential advantages of the fusion of multi focus and multispectral images. The increase in number, performance and reduced costs of sensors has led to a growth of the interest in sensor and data fusion for research purposes and in a number of application areas. Data fusion is widely adopted term describing the joint interpretation of data obtained from more than one input in order to generate enhanced information for decision making [1]. In image processing, fusion can be performed at the signal, feature and decision stages. Signal level fusion is performed on the pixels supplied in the visual information to form a single fused image. This type of fusion includes multi-focus image fusion, an area that has attracted much recent research investigation. Feature level fusion involves the fusion of features, labels or descriptor information that has been extracted from each input image. Decision-level fusion involves the fusion of high-level information extracted from the results of processing carried out on the separate images.

\section{RELATED WORK}

The aim of image data fusion is to obtain additional information by integrating the data obtained from individual sensors. Image data fusion increases the dimensionality of the data space, but often improves the quality of deduced information which is provided with less ambiguity and reduced system vulnerability. This area of fusion has been the focus of much research, but there is still the scope for further innovation [2].

Wavelet-based image fusion has been proposed for a multispectral palm print recognition system [3]. An infrared image was captured using a device that was able to adjust the images under different illumination conditions and the wavelet transform was applied to images with different types of channel to obtain a combined image. A competitive coding scheme was used as the matching method. This research has been extended so that the extraction and matching stages are formed of their own spectral bands of the multispectral palm image. A simple sum rule is then used on matching score level fusion. Feature-band selection based palm print recognition was proposed in which each single band is compared using the extracted statistical features and score-level fusion was 
adopted to determine the best combination from the alternatives available. A novel palm print verification method implemented by fusing palm images at a low level using the wavelet transform was proposed in [3]. The approach carried out image fusion in the wavelet domain, but image fusion using the discrete wavelet transform (DWT) requires many decomposition levels needing a large number of calculations and substantial memory capacity, and its implementation therefore takes considerable execution time. Although the DWT was able to improve recognition quality, it was still unable to provide a good representation of long edges in the fused result [4].

An image fusion algorithm that uses the energy of Laplacian (EOL) has been proposed, in which suitable input image regions are selected by determining their EOLs which is related to the portions of the image that are more in focus. A majority filter was used to ensure that regions in better focus influence the decisions taken regarding the selection of neighboring pixels and a guided filter generated an edge preserving process to reduce the block effects. The resulting image was shown to be de-blurred compared with the original images and exhibited an improved visual appearance compared to alternative approaches considered. Although this method was able to extract local and sharp intensity changes in images, it was also found that if one of the input images was of good clarity, the resulting image would often exhibit unpleasant visual artifacts in some regions [5].

The DCT has been widely used in image processing and pattern recognition because of its energy properties and high performance with respect to low order signals [6], with the DCT requiring less energy than an equivalent representation by the discrete wavelet transform (DWT). Several researchers have used DCT in image fusion as it is efficient due to the computational energy being much less than the transmission energy. DCT image fusion is mostly obtained by taking the average of all the coefficients in the images and so the fused result is likely to exhibit side effects such as blurring or blocking artifacts which reduce the quality of the image [4].

In this paper, a novel image fusion based on Multi Resolution DCT (MRDCT) and Frequency Partition DCT (FPDCT) are presented. DCT has been proven to perform well in image compression and is widely used in image preprocessing applications. A local feature-invariant method will be used to extract features from the vein images. By matching point-like features, a similarity distance measure can be calculated to perform classification and method was used to evaluate the recognition rate both before and after image fusion had been applied. Section III presents the process of the proposed method, involving the use of a subsection image database, the preprocessing stage, image fusion, feature extraction, mismatching point removal and feature matching, Section IV gives the experimental results and analysis and Section V provides the conclusions.

\section{METHODOLOGY}

This paper will focus on the effect that using DCT image fusion has on the recognition rate and extends previous work by the authors [7]. Fig. 1 shows the proposed method and it is divided into four steps. Images are acquired from the CASIA database and the preprocessing stage is similar to that used in previous work. The method proposed concentrates on image fusion techniques where the fused image is produced by integrating multiple image sources and is passed to the verification engine for performance evaluation [8].

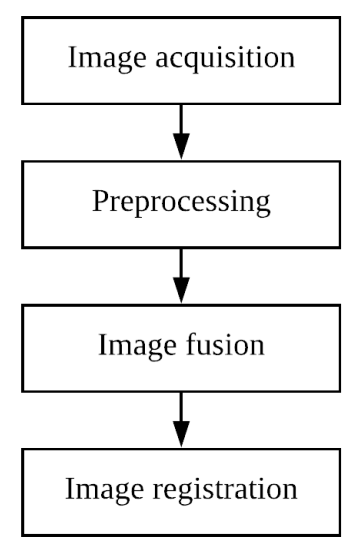

Figure 1. Flowchart of the proposed method.

\section{A. Discrete Cosine Transform (DCT) image fusion}

The DCT is used to express image data points at different frequencies by using the sum of cosine functions. The majority of DCT coefficients are focused on the low frequency area due to its energy compactness properties at different frequencies and the edges in the image contributes to high frequency coefficients [9].

The one dimensional (1D) DCT and inverse DCT (IDCT) are defined in equations (1) and (2) respectively. The DCT coefficient is obtained by using equation (1) and averaging all samples in the sequences and the remaining DCT coefficients obtained in both equations are real and provide weights for a set of discrete-time sinusoids. In order to extend to the 2D DCT, the 1D DCT formulae can be extended. The 2D DCT and 2D IDCT operate on the rows and columns of the image and are shown in equations (3) and (4) respectively. Fig. 2 shows the implementation of the 2D DCT, where column coefficients are determined first followed by the row coefficients second.

$X(k)=\alpha(k) \sum_{n=0}^{N-1} x(n) \cos \left(\frac{\pi(2 n+1) k}{2 N}\right), 0 \leq k \leq N-1(1)$

where $\alpha(k)=f(x)$.

$x(n)=\sum_{k=0}^{N-1} \alpha(k) X(k) \cos \left(\frac{\pi(2 n+1) k}{2 N}\right), 0 \leq n \leq N-1$

$X\left(k_{1}, k_{2}\right)=\alpha\left(k_{1}\right) \alpha\left(k_{2}\right)$.

$\sum_{n_{1}=0}^{N_{1}-1} \sum_{n_{2}=0}^{N_{2}-1} x\left(n_{1}, n_{2}\right) \cos \left(\frac{\pi\left(2 n_{1}+1\right) k_{1}}{2 N_{1}}\right) \cos \left(\frac{\pi\left(2 n_{2}+1\right) k_{2}}{2 N_{2}}\right)$

where $\alpha\left(k_{1}\right)$ and $\alpha\left(k_{2}\right)$ are similar to Equation (2). 


$$
\begin{aligned}
& X\left(k_{1}, k_{2}\right)=\sum_{n_{1}=0}^{N_{1}-1} \sum_{n_{2}=0}^{N_{2}-1} \alpha\left(k_{1}\right) \alpha\left(k_{2}\right) x\left(k_{1}, k_{2}\right) . \\
& \cos \left(\frac{\pi\left(2 n_{1}+1\right) k_{1}}{2 N_{1}}\right) \cos \left(\frac{\pi\left(2 n_{2}+1\right) k_{2}}{2 N_{2}}\right)
\end{aligned}
$$

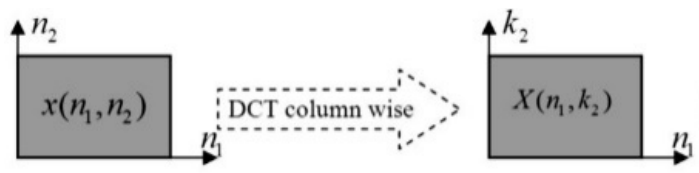

(a)

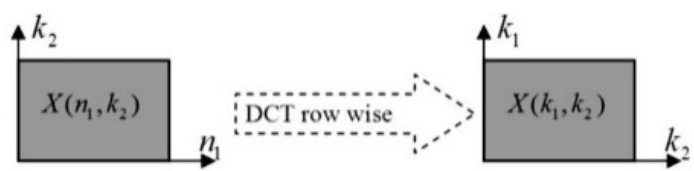

(b)

Figure 2. Computation of 2D DCT using separate (a) column and (b) row properties.

Fig. 3 shows a simple image fusion process using the DCT and the IDCT. Here, an input image is divided into nonoverlapping blocks of size 8x8 and then transformed into DCT coefficients. A fusion rule is then applied in the DCT coefficient of each block. Consecutive 8x8 DCT domain blocks are then converted into the spatial domain by applying IDCT [10].

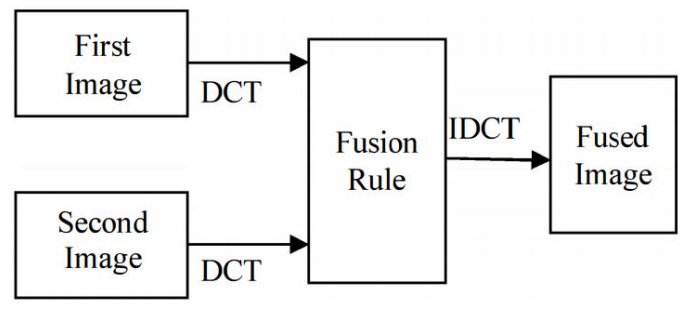

Figure 3. Image fusion process using DCT.

\section{B. Frequency Partition Discrete Cosine Transform (FPDCT)}

The FPDCT will partition the DCT coefficients into low frequency (LF) and high frequency (HF) regions. Partition is carried out according to the partition factor $\mathrm{f}$ by using the energy compaction property of DCT coefficients. The partition factor $\mathrm{f}$ is a value in the range 0 to 1 with a resolution 0.1 , thereby defining 10 distinctive levels. The image is down sampled and by selecting the frequency level according to the pixel intensity, the image is fused according to Equation (5). The fused image is then up sampled to its original size using the IDCT. Fig. 4 shows the partition method carried out to partition DCT coefficients into the LF and HF regions [11].

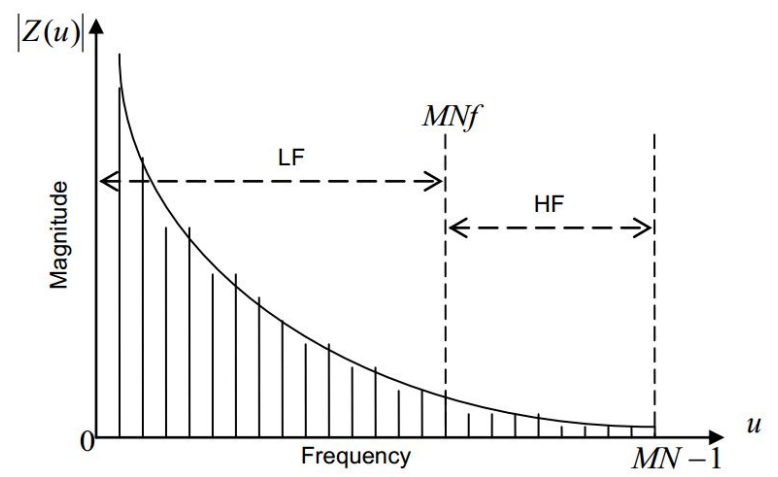

Figure 4. Separation of LF and HF coefficients.

\section{Multi-resolution DCT (MRDCT)}

In MRDCT, the DCT coefficients are filtered by low-pass and high-pass finite impulse filters (FIRs). The number of DCT coefficients will be separated into two separate halves and each half is filtered by both high-pass and low-pass filters. The output filter is then decimated by a factor of two to achieve the first-level decomposition. Second-level decomposition repeats the operations of the first level by using the decimated high-pass and low-pass coefficients. Further levels of decomposition are successively implemented by repeating the procedure in low-pass and high-pass filters [10]. Fig. 5 shows the outcome as the DCT coefficients are processed by low-pass and high-pass filters at each level. MRDCT has characteristics similar to the wavelet transform in the manner in which the operations are organized and the representations used for its coefficients [6].

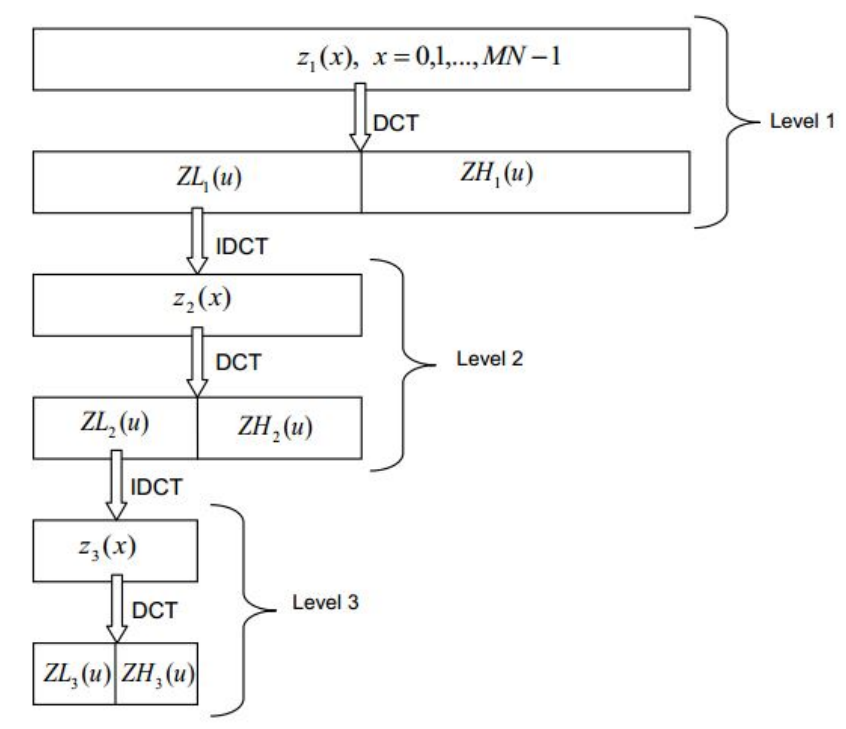

Figure 5. Multi resolution image analysis using DCT. 


\section{RESULTS AND DISCUSSION}

\section{A. Experimental setup}

This experiments presented in this section evaluate the impact of performing image fusion on the palm vein recognition rate. The work has used the CASIA database that contains data from 100 different subjects each of which has provided six images. In this experiment, five images from 100 subjects were used for testing and there were a total of 356400 outer and 3600 inner point to point matches. Equal Error Rate (EER) was plotted for all the different sample variations.

\section{B. Image fusion techniques}

Fig. 6 to 9 show the results from four fusion techniques, namely FPDCT, MRDCT, wavelet and EOL. The fusion techniques were all applied at wavelengths of 700nm, 850nm and $940 \mathrm{~nm}$ to example palm vein images. In each of the figures, images (a) to (c) are the original images at the three wavelength spectrum of $700 \mathrm{~nm}, 850 \mathrm{~nm}$ and $940 \mathrm{~nm}$ respectively, images $(\mathrm{d})$ to $(\mathrm{g})$ show the fusion techniques implemented respectively between the $700 \mathrm{~nm}$ with $850 \mathrm{~nm}$ images, the $700 \mathrm{~nm}$ and $940 \mathrm{~nm}$ images, the $850 \mathrm{~nm}$ and $940 \mathrm{~nm}$ images and between all three images.

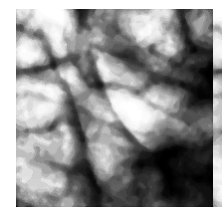

(a)

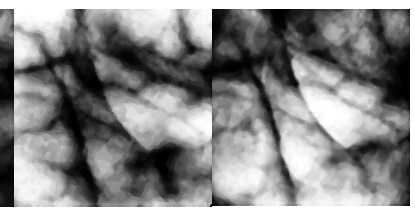

(b)

(c)

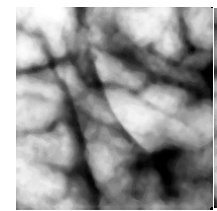

(d) (e) (f)

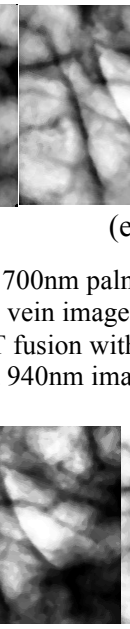

(a)

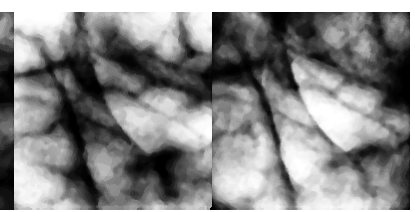

(b) (c)

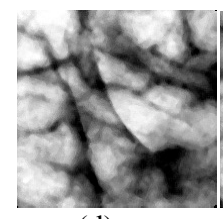

(d)

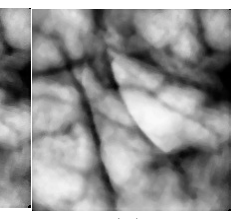

(g)
Figure 6. (a) $700 \mathrm{~nm}$ palm vein image. (b) $850 \mathrm{~nm}$ palm vein image. (c) $940 \mathrm{~nm}$ palm vein image. (d) FPDCT fusion with $700 \mathrm{~nm}$ and $850 \mathrm{~nm}$ mage. (e) FPDCT fusion with 700nm and 940nm image. (f) FPDCT fusion with $850 \mathrm{~nm}$ and $940 \mathrm{~nm}$ image. (g) FPDCT fusion with all image above.

Figure 7. (a) $700 \mathrm{~nm}$ palm vein image. (b) $850 \mathrm{~nm}$ palm vein image. (c) $940 \mathrm{~nm}$ palm vein image. (d) MRDCT fusion with $700 \mathrm{~nm}$ and $850 \mathrm{~nm}$ image. (e) MRDCT fusion with 700nm and 940nm image. (f) MRDCT fusion with $850 \mathrm{~nm}$ and $940 \mathrm{~nm}$ image. (g) MRDCT fusion with all image above.

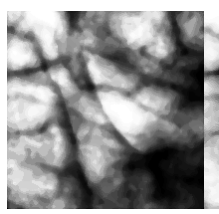

(a)

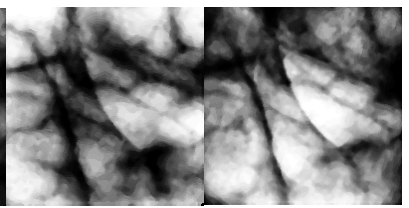

(b) (c)

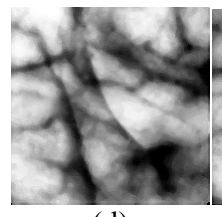

(d)

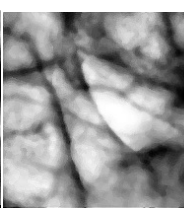

(e)

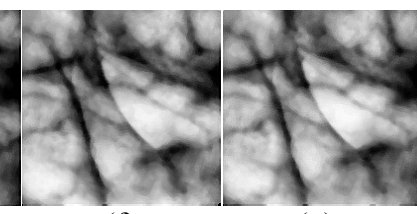

(f) (g)
Figure 8. (a) $700 \mathrm{~nm}$ palm vein image. (b) $850 \mathrm{~nm}$ palm vein image. (c) $940 \mathrm{~nm}$ palm vein image. (d) Wavelet fusion with $700 \mathrm{~nm}$ and $850 \mathrm{~nm}$ image. (e) Wavelet fusion with 700nm and 940nm image. (f) Wavelet fusion with $850 \mathrm{~nm}$ and $940 \mathrm{~nm}$ image. (g) Wavelet fusion with all image above.

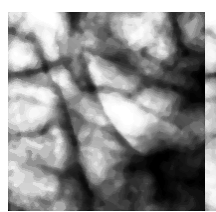

(a)

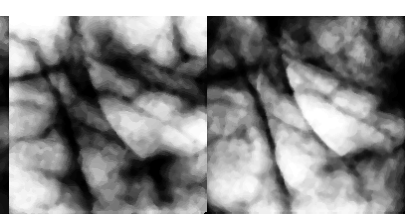

(b) (c)

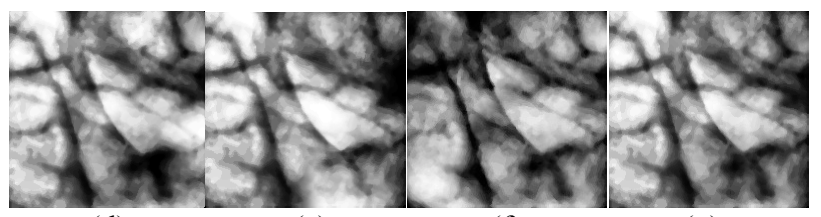

(d) (e) (f) (g)
Figure 9. (a) $700 \mathrm{~nm}$ palm vein image. (b) $850 \mathrm{~nm}$ palm vein image. (c) $940 \mathrm{~nm}$ palm vein image. (d) EOL fusion with $700 \mathrm{~nm}$ and $850 \mathrm{~nm}$ image. (e) EOL fusion with $700 \mathrm{~nm}$ and 940nm image. (f) EOL fusion with $850 \mathrm{~nm}$ and $940 \mathrm{~nm}$ image. (g) EOL fusion with all image above.

For all the fusion images obtained for all four fusion techniques, it can be clearly seen that right hand side of the image produced a better visual effect. The original image with wavelength $850 \mathrm{~nm}$ has a better vein pattern on the right hand side while the $940 \mathrm{~nm}$ wavelength and $700 \mathrm{~nm}$ wavelength images have a blurred vein pattern. The dark area on the righthand side has become de-blurred once the fusion techniques have been applied. This can be assumed to be because the fusion techniques have enhanced the darkness area by fusion with the second image that has no area of darkness on the right hand side. Some of the areas on the left hand side of the image have also been enhanced. The fusion images show the finer details of vein patterns and while a number of the darker areas have also been enhanced by fusion with the first image. An explanation is that the fusion techniques are able to recover the information from other images in order to replace the darkened areas in another of the fused images. A similar trend can be found for other image fusion techniques. Hence, it appears that image fusion enhances and preserves information without creating additional unwanted noise. However, in EOL and wavelet based image fusion, the fusion image is able to obtain the vein patterns but the pattern shows some artifacts. 


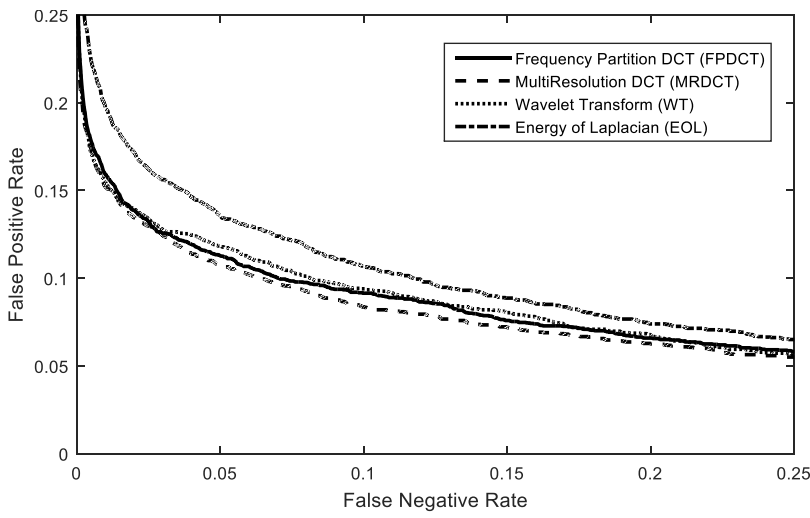

(a)

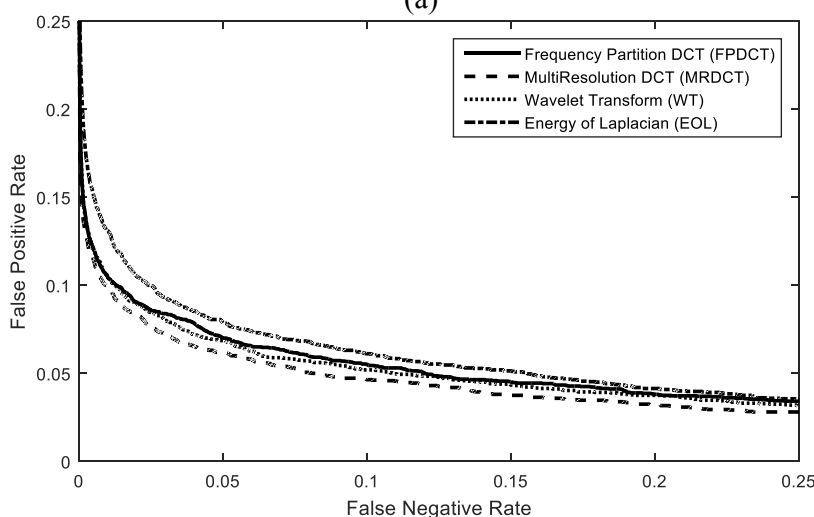

(c)

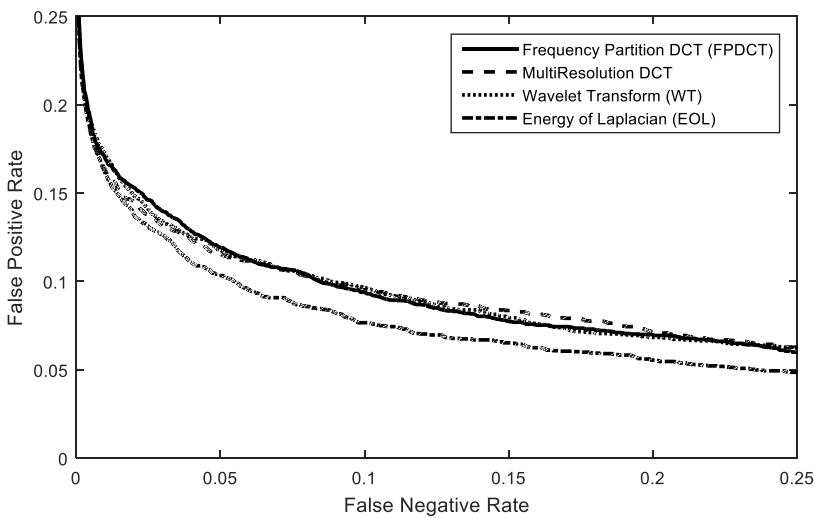

(b)

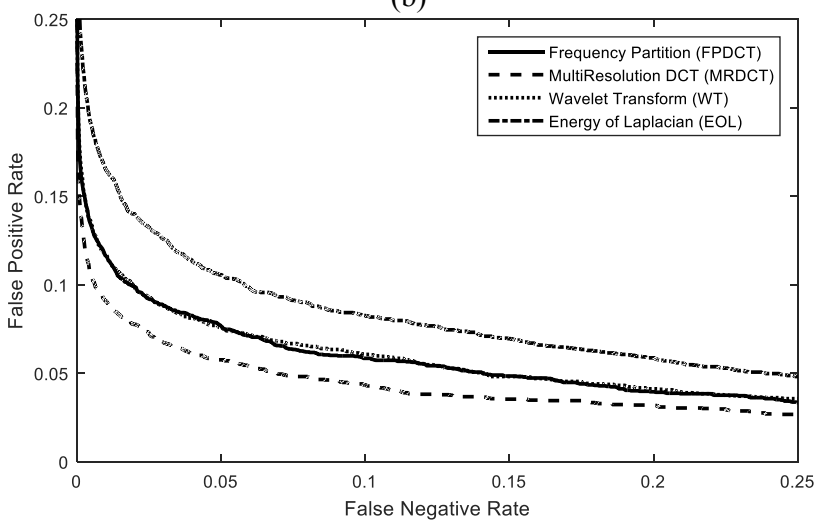

(d)

Figure 10. Equal Error Rate of all fusion techniques (a) Fusion in 700nm and 850nm, (b) Fusion in 700nm and 940nm, (c) Fusion in 850nm and 940nm, (d) Fusion in $700 \mathrm{~nm}, 850 \mathrm{~nm}$ and $940 \mathrm{~nm}$ image.

Fig. 10 shows the equal error rate (EER) rates obtained when pairs of image fusion wavelengths have been combined and where all the three wavelengths have been combined. MRDCT achieved the best performance when $700 \mathrm{~nm}$ with $850 \mathrm{~nm}$ images were combined and when $850 \mathrm{~nm}$ with $940 \mathrm{~nm}$ images were combined. EOL achieved the best performance for fusion of the $700 \mathrm{~nm}$ with $940 \mathrm{~nm}$ images. In general, it can be seen that the results obtained from EER improve once the image fusion has been applied as well as there being an increase in the number of matching points. It can be concluded that as the number of matching point is increased, the matching percentage on the image will also increase and so the possibility of a genuine match will be greater. In each of the FPDCT, the MRDCT and the wavelet transform, the EER rate is around $9.5 \%$ and this decreases only slightly by $0.5 \%$ compared to the results obtained from the $700 \mathrm{~nm}$ with $850 \mathrm{~nm}$ fusion. However, EOL achieved the result that improved by $2 \%$ for the $700 \mathrm{~nm}$ with $940 \mathrm{~nm}$ fusion compared with the $700 \mathrm{~nm}$ with $850 \mathrm{~nm}$ image fusion.

Table I and II shows the EER and area under curve (AUC) that result from the use of the different image fusion techniques. However, the performance of the fusion of the $850 \mathrm{~nm}$ with $940 \mathrm{~nm}$ images is very similar to that obtained from the combination of the three images. The performance shows a degradation between $0.5 \%$ and $1.5 \%$ when all images are fused, perhaps because the fusion is adversely affected by the fusion with the 700nm image. In MRDCT, a different result is apparent in which the results are improved by $0.3 \%$ compared to the $850 \mathrm{~nm}$ with $940 \mathrm{~nm}$ fusion. By comparing the AUC curve, the classification is improved by using MRDCT with the fusion of the three images and this achieved the highest classification rate of $98.11 \%$. It can be seen that MRDCT is the most suitable technique for use in palm vein recognition when all three types of image are fused.

TABLE I. EQUAL ERROR RATE IN 700NM,850NM AND 940NM IMAGE

\begin{tabular}{|l|c|c|c|c|}
\hline Method & $\begin{array}{c}700 \mathrm{~nm} \\
\text { and } \\
\mathbf{8 5 0 n m}\end{array}$ & $\begin{array}{c}\mathbf{7 0 0 n m} \\
\text { and } \\
\mathbf{9 4 0 n m}\end{array}$ & $\begin{array}{c}\mathbf{8 5 0 n m} \\
\text { and } \\
\mathbf{9 4 0 n m}\end{array}$ & $\begin{array}{c}\mathbf{7 0 0 n m} \\
\mathbf{8 5 0 n m} \\
\text { and } \\
\mathbf{9 4 0 n m}\end{array}$ \\
\hline MRDCT & 8.83 & 9.65 & 5.83 & 5.53 \\
\hline FPDCT & 9.33 & 9.56 & 6.47 & 6.76 \\
\hline Wavelet & 9.47 & 9.75 & 6.16 & 6.83 \\
\hline EOL & 10.47 & 8.47 & 6.99 & 8.64 \\
\hline
\end{tabular}


TABLE II. AREA UNDER CURVE (AUC) IN 700NM,850NM AND 940NM IMAGE

\begin{tabular}{|l|c|c|c|c|}
\hline Method & $\begin{array}{c}\mathbf{7 0 0 n m} \\
\text { and } \\
\mathbf{8 5 0 n m}\end{array}$ & $\begin{array}{c}\mathbf{7 0 0 n m} \\
\text { and } \\
\mathbf{9 4 0 n m}\end{array}$ & $\begin{array}{c}\mathbf{8 5 0 n m} \\
\text { and } \\
\mathbf{9 4 0 n m}\end{array}$ & $\begin{array}{c}\mathbf{7 0 0 n m} \\
\mathbf{8 5 0 n m} \\
\text { and } \\
\mathbf{9 4 0 n m}\end{array}$ \\
\hline MRDCT & 96.23 & 95.77 & 97.91 & 98.11 \\
\hline FPDCT & 95.95 & 95.90 & 97.61 & 97.57 \\
\hline Wavelet & 95.91 & 95.73 & 97.68 & 97.50 \\
\hline EOL & 95.36 & 96.70 & 97.43 & 96.55 \\
\hline
\end{tabular}

\section{CONCLUSION}

Image fusion has a major impact on the recognition performance in the palm vein recognition system. This paper has focused on the application of image fusion techniques that affect the recognition rate. Image fusion techniques are able to recover vein information with a reduced presence of noise. Vein patterns become more obvious and it becomes possible to observe them by visual inspection. Although several DCT fusion methods have been introduced, DCT has been shown to provide good results for image fusion when implementing a palm vein recognition system.

MRDCT achieved the best performance rate, the lowest EER rate of $5.53 \%$ and $98.11 \%$ in the AUC curve. MRDCT outperformed the other fusion techniques in both dual and triple combinations of wavelengths for image fusion. MRDCT provided improvements of almost $9 \%$ for a $700 \mathrm{~nm}$ image, $7 \%$ for an $850 \mathrm{~nm}$ image and $6 \%$ for a $940 \mathrm{~nm}$ image. This method is able to preserve well the detailed information without producing any unpleasing effects on the image.

\section{ACKNOWLEDGMENT}

This work was supported by Universiti Malaysia Pahang and funded by the Ministry of Higher Education Malaysia under Fundamental Research Grant Scheme (FRGS) RDU160108.

\section{REFERENCES}

[1] D.L. Hall and J. Llinas, "An introduction to multisensor data fusion," Proc. IEEE, vol. 85, no. 1, pp. 6-23, 1997.

[2] Dong Jiang, Dafang Zhuang, Yaohuan Huang and Jinying Fu, "Survey of Multispectral Image Fusion Techniques in Remote Sensing Applications, Image Fusion and Its Applications", IntechOpen, 2011.

[3] D.Han, Z.Guo, and D.Zhang, "Multispectral palmprint recognition using wavelet-based image fusion," Int. Conf. Signal Process (ICSP), pp. 2074-2077, 2008.

[4] Y.A.Victy and R.Amutha, "Discrete Cosine Transform based fusion of multi-focus images for visual sensor networks," Signal Processing, vol. 95, pp. 161-170, 2014.

[5] K.Zhan, J.Teng, Q.Li, and J.Shi, "A novel explicit multi-focus image fusion method A Novel Explicit Multi-focus Image Fusion Method," Journal of Information Hiding and Multimedia Signal Processing, vol 6, no. 3, 2015.

[6] B.K. Shreyamsha Kumar, M.N.S. Swamy, and M. Omair Ahmad "Multiresolution DCT Decomposition for Multifocus Image Fusion", IEEE Canadian Conference on Electrical and Computer Engineering (CCECE), pp. 1-4, 2013

[7] S.C. Soh, M.Z. Ibrahim, M.B. Yakno, and D.J. Mulvaney, "Palm Vein Recognition Using Scale Invariant Feature Transform with RANSAC Mismatching Removal," in IT Convergence and Security 2017, Springer, 2018, pp. 202-209.

[8] R. Bhokare, D. Sale, M.A. Joshi, and M.S.Gaikwad, "Multispectral Palm Image Fusion : A Critical Review," vol. 2, no. 6, pp. 2159-2164, 2013.

[9] V.P.S. Naidu, "Novel Image Fusion Techniques using DCT," vol. 5, no. 1, pp. 1-18, 2013.

[10] V.P.S. Naidu, "Discrete Cosine Transform based Image Fusion Techniques," Journal of Communication, Navigation and Signal Processing, vol 1. no. 1, pp. 35-45, 2012.

[11] V.Kaur and J.Kaur, "Frequency Partioning Based Image Fusion for CCTV," International Journal of Computer Science and Information Technologies, vol. 6, no. 4, pp. 3968-3972, 2015. 